WPSReview International on Sustainable

Housing and Urban Renewal (RI-SHUR)

\title{
NUEVO MODELO DE CENTRO DE TRANSFORMACIÓN SUBTERRÁNEO INTELIGENTE, ADAPTADO A LAS SMART CITIES Y AL CAMBIO CLIMÁTICO
}

\author{
Inés Aragüez del Corral \\ Moisés Garrido Martín \\ Montajes Eléctricos Cuerva S.L.
}

Artículo Recibido: 28/04/2018

Artículo Aceptado: 14/09/2018

\section{Resumen}

Las smart grids se enfrentan al reto de disponer de un nodo inteligente que permita la gestión de la red de distribución. Este no puede ser otro que los centros de transformación, como nodo energético al que llegan las redes de media tensión de las subestaciones y de donde parten las redes en baja tensión que alimentan a los consumidores, y como nodo en la arquitectura de comunicaciones necesaria para el control inteligente de la red.

Hay que destacar que es fundamental que estos elementos consigan una mayor fiabilidad, seguridad y una mejor continuidad de suministro en caso de catástrofes (inundaciones, incendios, terremotos, ...) No se puede olvidar que en estas situaciones de emergencia, conseguir que la red eléctrica de distribución siga funcionando, y que lo haga de forma inteligente, es fundamental para poder alimentar los servicios críticos, como pueden ser comunicaciones, centros sanitarios, sistemas de bombeo,... ayudando a conformar lo que se denomina "ciudades resilientes".

También es importante considerar que la ubicación de nuevos centros de transformación en núcleos urbanos consolidados es cada vez más difícil. Este efecto se va a ver acentuado aún más si se prevé la incorporación del vehículo eléctrico como medio habitual de movilidad urbana, ya que esto va a suponer un aumento considerable de la potencia instalada. 


\title{
WPSReview International on Sustainable
}

\author{
Housing and Urban Renewal (RI-SHUR)
}

Se propone un nuevo modelo de centro de transformación subterráneo e inteligente que, con un diseño innovador, sea capaz de resolver estos problemas. Este modelo propuesto cuenta con una envolvente metálica cilíndrica que le confiere una mayor resistencia ante grandes esfuerzos exteriores, manteniendo su estanqueidad ante inundaciones y las acciones propias de movimientos sísmicos. En su interior se dispondrá toda la aparamenta, con accionamientos motorizados, dispondrá de relés de protección y sensores de parámetros de control. Todo el sistema se controla desde una centralita que se sitúa en un cofre estanco y resistente exterior. Desde esta centralita se podrán gestionar todos los accionamientos, comprobar el estado de conexión y desconexión de cada uno de éstos y conocer los parámetros de funcionamiento de los equipos. También, la centralita permitirá almacenar un histórico de maniobras realizadas y accesos permitidos mediante sistema big data con almacenamiento de información en la nube. Este sistema también permitirá la posibilidad de una telegestión desde un centro de control o a través de una APP (aplicación) para dispositivos móviles, permitiendo la incorporación de este equipo en otro de gestión integral inteligente o smart grids. Además, va a permitir una mejor gestión del mantenimiento y una explotación de la red más eficiente.

Para solventar la evacuación de calor de la cuba del transformador, se modifica el diseño convencional de aletas adosadas a la cuba por un nuevo diseño basado en instalar intercambiadores en el exterior y mediante unos colectores en la cuba que permitirán hacer circular el aceite refrigerante mediante una bomba. Para una total mimetización del centro, este intercambiador se instalará sustituyendo parte del bordillo del acerado. De esta forma, el centro queda totalmente estanco y resistente a cualquier tipo de inundación, tsunami o avenida. Además, este sistema de refrigeración, más eficiente, unido a la ausencia de rejillas, va a aumentar la durabilidad de los componentes y su vida útil.

Se concluye que el uso de este innovador modelo de centro de transformación va a conseguir que las infraestructuras eléctricas de las ciudades seas más resistentes y operativas, permitiendo afrontar los grandes retos del futuro que van a traer la revolución digital, los vehículos eléctricos y el cambio climático.

\section{Abstract}

Smart grids face the challenge of having an intelligent node that allows the management of distribution network. This one can not be other than transformer stations, as an energetic node where high voltage grid get to, and where distribution grid come out in low voltage for consumers, and as a node in the communications architecture, necessary for the intelligent control of the network.

It should be noted that it is important that these energy and communication nodes achieve greater reliability, security and a better continuity of supply in case of catastrophes (floods, fires, earthquakes,...). It can not be forgotten that in these emergency situations, getting the distribution grid working is essential to be able to 


\section{WPSReview International on Sustainable \\ Housing and Urban Renewal (RI-SHUR)}

supply critical services, such as communications, hospitals, pumping systems, ... helping to create what is called "resilient cities".

It is also important to consider that the location of new transformer stations in consolidated urban centres is increasingly difficult. This effect will be accentuated even more if the incorporation of the electric vehicle as a usual means of urban mobility is taken into account, since this will mean an increase in the installed power of the distribution grid.

A new model of underground smart transformer station is proposed, which, with an innovative design, is capable of solving these problems. The proposed model has a cylindrical metallic envelope, giving it a greater resistance to external forces while keeping its watertightness against floods and forces characteristic of seismic movements. Within the inside, all the switchgear is available, with motorized drives. It will have protection relays and sensors to control parameters. All the system is managed by a control unit which is located in a tight and resistant exterior box. From this control unit, all the elements can be managed, checking the connection status of each one and knowing the operating parameters of the equipment. Also, the control unit will enable to store a data history of performed operations and granted accesses by big data and Cloud Computing technologies. The system will also allow the possibility of a remote management from a control station or through an APP (application) system for mobile devices, allowing the incorporation of this equipment in another intelligent integrated one or smart grids. In addition, it will allow a better maintenance management and a more efficient operation of the network.

With the purpose of solving the heat evacuation of the transformer tank, the conventional fin design of the tank is modified by a new design based on installing exchangers on the outside and by means of collectors in the tank that will allow the refrigerant oil to circulate with a pump. In order to make the station invisible, this exchanger will be installed replacing part of the curb on the sidewalk. In this way, the station is totally sealed and resistant to any type of flood or tsunami. In addition, this cooling system, more efficient, and an envelope without openings, will increase durability of components and their useful life.

It is hereby concluded that the use of this innovative transformer station model will make electrical infrastructures of cities more resistant and operational, enabling them to face the great challenges of the future brought by the digital revolution, electric vehicles and climate change.

\section{Palabras clave}

smart cities; smart grids; red de distribución; resiliencia urbana; cambio climático; centro de transformación 


\section{WPSReview International on Sustainable \\ Housing and Urban Renewal (RI-SHUR)}

\section{Key Words}

smart cities; smart grids; electrical distribution grid; urban resilience; climate change; transformer station

\section{1.- Introducción}

Los centros de transformación deben albergar los elementos de control automático necesarios para permitir que, desde ellos, se pueda interactuar en las redes de alta y baja tensión. Este sistema así creado va a conseguir una adecuada gestión de las redes de distribución, permitiendo que éstas conformen una smart grid que proporcione nodos de energía y comunicación que puedan ser controlados mediante un sistema inteligente de la red. Los centros que se pueden encontrar en el mercado actual, por su geometría, materiales de construcción y el sistema de ventilación empleado, son muy vulnerables en caso de inundaciones, tsunamis, terremotos, etc. La presente propuesta trata de, olvidando los criterios de diseño existentes y de una manera objetiva, estudiar la implantación de un nuevo modelo que propicie una mayor seguridad y una mejor continuidad de suministro en caso de catástrofes naturales y efectos del cambio climático (inundaciones, incendios, terremotos,...), dotándolo de sistemas que lo hagan resistente ante estas situaciones (resiliencia urbana). Tampoco se puede obviar que en las grandes ciudades es muy difícil ubicar nuevos centros de transformación, y aún más si se prevé la incorporación del vehículo eléctrico como medio habitual de movilidad urbana, con su correspondiente aumento de potencia instalada, por lo que es importante adoptar nuevos diseños que permitan disponer estos nuevos centros en aquellos lugares donde sea importante el impacto visual y en donde no exista suelo libre para ubicar las casetas tradicionales.

\section{2.- Metodología}

\section{2- Retos para el futuro}

2.2.1. Resiliencia urbana ante catástrofes

El cambio climático nos aboca a tener que prever que las ciudades se enfrenten a una climatología más adversa de lo usual, además de las catástrofes habituales de origen geológicos (terremotos, tsunamis,...), hay que añadir inundaciones, desbordamientos de ríos, grandes incendios forestales, aumentos del nivel del mar, etc.

Los centros de transformación convencionales son muy vulnerables ante este tipo de siniestros, quedando inutilizados y provocando grandes apagones que se prolongan en el tiempo por la dificultad que entraña sus reparaciones. Las autoridades locales dan cada vez mayor importancia a que, en caso de siniestros, la red eléctrica siga funcionando para conseguir que los equipos básicos y urgentes se mantengan operativos (servicios sanitarios, hospitales, comunicaciones, abastecimiento de agua, sistemas de bombeo, y un largo etcétera) (Resccue, 2018). De esta manera, se va a ayudar a conseguir que la recuperación ante cualquier catástrofe sea más rápida y, 


\section{WPSReview International on Sustainable \\ Housing and Urban Renewal (RI-SHUR)}

sobre todo, reducir el número de víctimas. "Invertir un dólar en prevención de desastres tiene un retorno de 130 dólares" (El Confidencial, 2013) ${ }^{1}$.

\subsubsection{Ciudades con climatología o condiciones adversas}

En zonas desérticas la existencia de tormentas de arena es una problemática que merece una mención especial, ya que, por su gran cantidad y su pequeña granulometría va a penetrar por todos los huecos de los equipos colapsando el sistema en muy poco tiempo.

También hay que destacar que en aquellos lugares en los que la temperatura ambiental es muy alta, la refrigeración del transformador mediante convección natural se dificulta enormemente por el pequeño salto térmico existente entre la temperatura exterior y la temperatura de trabajo de la máquina. Esto provoca problemas en el funcionamiento y disminuye en gran medida el rendimiento del transformador. (Aragüez, 2016b)

\subsubsection{Integración de la red de distribución en sistemas smart grids}

Cada vez más se están configurando lo que se ha dado en llamar ciudades inteligentes (smart cities), y como figura fundamental, las redes eléctricas inteligentes (smart grids). Para poder configurar redes inteligentes es necesario disponer elementos que puedan ser gestionados mediante un sistema programable. Este sistema ha de recoger valores de sensores existentes y poder mandar señales de actuación de acuerdo con los algoritmos planteados. Los centros de transformación son los elementos donde se pueden implementar estos sistemas de control, y propiciar así una adecuada integración de la red en el conjunto de una gestión más general.

\subsubsection{Integración de los centros de transformación en el entorno urbano}

La ubicación de nuevos centros de transformación en las ciudades plantea serios problemas urbanísticos, ya que situar centros de superficie va a chocar con las figuras de protección de centros históricos y de integración urbana e incluso, posibles problemas de contaminación por campos eléctricos y magnéticos o ruidos y vibraciones. Pensemos en centros turísticos, de alto valor comercial, históricos, de alta densidad de población, etc.

Si se plantea el uso de locales comerciales, resulta inviable por ser prohibitivo, dado su alto valor económico.

La ubicación de centros de transformación subterráneos convencionales no es una solución completa ya que hay que disponer de antiestéticas chimeneas de ventilación equipadas con rejillas de ventilación.

\footnotetext{
${ }^{1}$ https://www.elconfidencial.com/ultima-hora-en-vivo/2013-12-17/invertir-un-dolar-en-prevencion-dedesastres-tiene-un-retorno-de-130-dolares_126327/
}

WPS RI-SHUR, no-7-8, 2018, vol.1, ISSN: 2387-1768 


\section{WPSReview International on Sustainable \\ Housing and Urban Renewal (RI-SHUR)}

\subsubsection{Implantación de los vehículos eléctricos}

La paulatina incorporación de los vehículos eléctricos presenta un horizonte en el que, a largo plazo, entorno a un $40 \%$ del parque automovilístico va a ser de tracción eléctrica y, por lo tanto, se va a demandar una potencia instalada en redes de distribución del orden de un $35 \%$ superior a la existente. Esto va a conllevar la necesidad de instalar nuevos centros de transformación, sobre todo, en zonas de mayor densidad urbana. (Aragüez, 2016b) (REE, 2018)

\subsection{Mayor seguridad laboral}

La manipulación directa de los interruptores existentes en el centro de transformación (en alta y en baja tensión) es una práctica habitual y cotidiana pero que ha provocado multitud de accidentes, ya que si existe un cortocircuito no detectado puede ocasionar una proyección de metal fundido que provoque graves quemaduras en el operario.

Por otro lado, la manipulación directa del aparellaje en alta tensión puede provocar la electrocución por contacto accidental del operario con alguna zona en tensión en el espacio confinado del centro de transformación.

En caso de que se esté reparando un tramo de red, puede ocurrir que, por error, se conecte dicho tramo pudiendo provocar un accidente en las personas que en ese instante están trabajando.

\subsection{Seguridad ante incendios}

Puede ocurrir que un fallo en el bobinado del transformador o una deficiente refrigeración provoque un incendio de la máquina que, teniendo en cuenta el gran volumen de aceite presente en la cuba, puede tener una magnitud importante. Este riesgo se agrava si el centro de transformación ocupa un local de un edificio de viviendas u oficinas. En ese caso, al problema propio de las llamas, hay que unir el hecho de la gran cantidad de humo que emite este tipo de incendios.

\subsubsection{Presencia de polvo, insectos y roedores}

La presencia de rejillas de ventilación va a hacer que en el interior de los centros de transformación se acumule polvo sobre los elementos en tensión. Este polvo en condiciones de humedad ambiental alta puede volverse conductor y provocar averías.

Por otro lado, estas rejillas van a ser el lugar por donde penetren animales que aniden en el interior del centro, e incluso, que deterioren los aislamientos, provocando, igual que en el caso anterior, graves averías. 


\section{WPSReview International on Sustainable \\ Housing and Urban Renewal (RI-SHUR)}

\section{3.- Resultados y Conclusiones}

\subsection{Propuesta de solución}

En este artículo se plantea la implantación de un nuevo modelo de centro de transformación, analizando pormenorizadamente las ventajas que éste va a suponer sobre los convencionales. En este modelo propuesto (Aragüez, 2016a), de tipo subterráneo y totalmente estanco, todos los equipos se han diseñado, calculado y simulado mediante software específicos (Aragüez, 2016b), usándose estándares europeos en general, y la normativa española, en particular (RD 337/2014). En el caso de que este modelo se implante en otros países, se deberá estudiar la adaptación a la normativa local correspondiente.

\subsubsection{Envolvente}

La envolvente consiste en un cilindro de acero con virola a ambos lados, como se ve en la figura 1. Se escoge esta tipología geométrica ya que presenta dos grandes ventajas:

- El cilindro es una figura geométrica que se comporta mejor ante los posibles empujes del terreno.

- La forma constructiva, por medio del curvado de chapa, hace que sólo presente un único cordón de soldadura en la generatriz del cilindro (aparte de los necesarios para los casquetes extremos). De esta manera, los posibles puntos de corrosión se reducen al mínimo.

Este cilindro se protege mediante revestimiento exterior de PRFV (poliéster reforzado con fibra de vidrio), para evitar problemas de corrosión, y se dota de dos tapas para el paso de personas (zona de alta tensión y zona de baja tensión) y una tercera, de mayor dimensión, para el montaje y desmontaje del aparellaje. Los extremos se sellan con tapas atornilladas a las virolas y juntas elásticas. El conjunto se fija sólidamente a una solera armada que se dispone en el fondo de la excavación. 

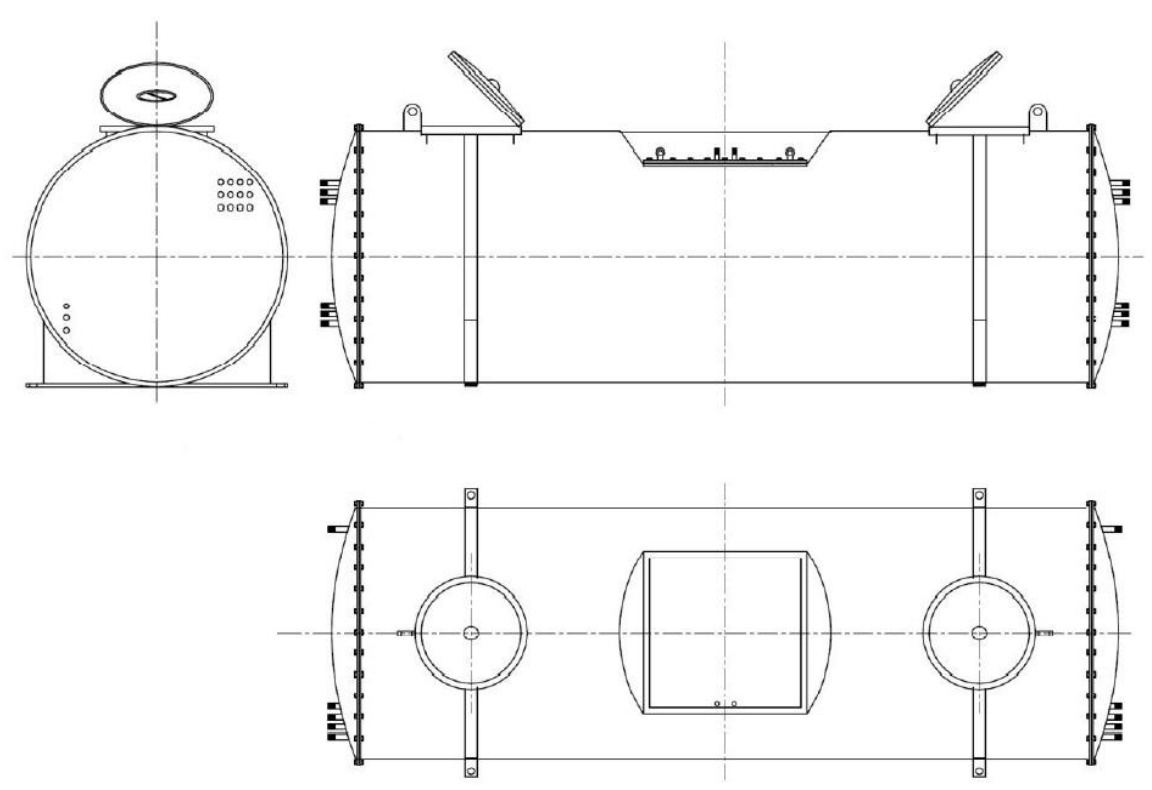

Figura 1: Detalle de envolvente del centro de transformación

\subsubsection{Aparellaje}

Se concibe este centro de transformación con la mínima necesidad de acceder a su interior, para lo cual se realizan las siguientes modificaciones en los aparellajes usuales (ABB, 2004):

- Se dotan de motorización de conexión y desconexión a todos los interruptores de las celdas de alta tensión (Capella, 2000). Los elementos se calculan de acuerdo a la reglamentación correspondiente. (RD 223/2008).

- La protección del transformador se realizará mediante relé magnético y térmico, regulable según potencia del transformador, que accionará el interruptor motorizado correspondiente (ABB, 2004).

- Las salidas en baja tensión se dotarán de interruptores automáticos en caja moldeada con motor de conexión y desconexión. Su calibre y regulación se ajustará a la sección de conductor empleado. Los elementos se calculan de acuerdo a la reglamentación correspondiente. (RD 842/2002).

- La conexión transformador - cuadro de baja tensión se realiza mediante pletinas, suprimiéndose los haces de conductores de enlace habituales.

En la figura 2 se comprueba la disposición de estos elementos. 
WPSReview International on Sustainable

Housing and Urban Renewal (RI-SHUR)

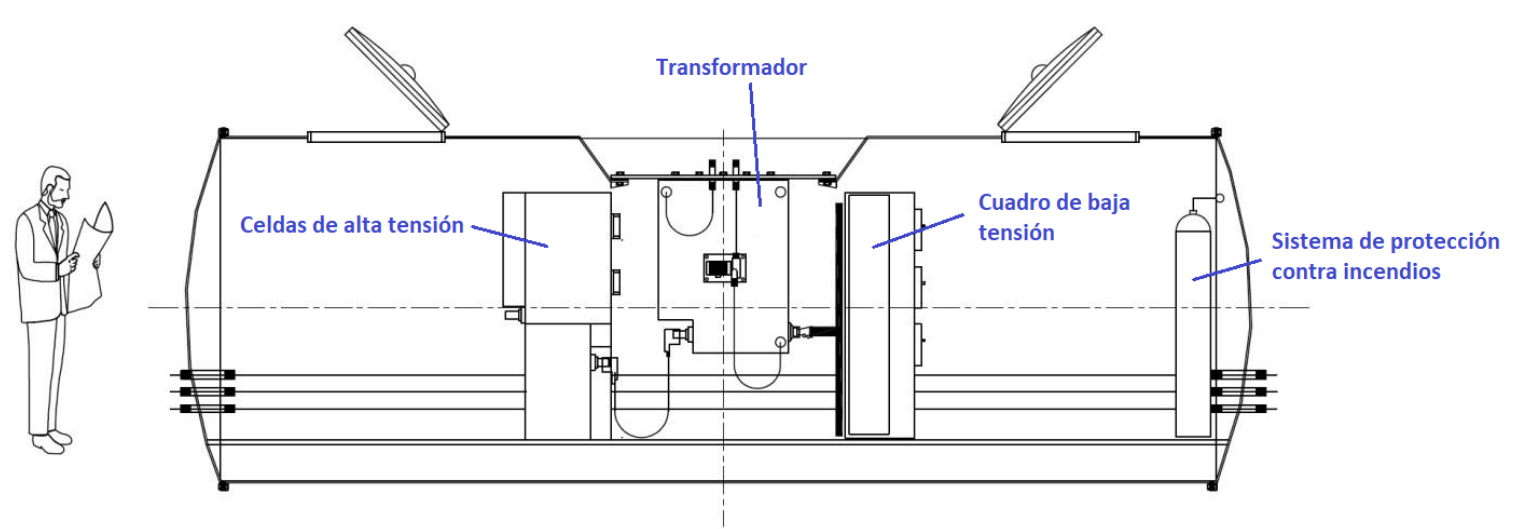

Figura 2: Sección longitudinal del centro de transformación propuesto

\subsubsection{Transformador}

Sobre el transformador estándar (Avelino, 1998) (Schneider Electric, 2014) se realizarán las siguientes modificaciones (UNE-EN 60076-1, 2013):

1. Supresión de las aletas de ventilación y ruedas de transporte

2. Instalación y girado de conos de conexión de alta tensión y giro de $90^{\circ}$ de las pletinas de salida en baja tensión.

3. Colocación de colectores interiores para la recirculación externa del aceite refrigerante

4. Colocación de vainas para instalación de detectores de temperatura y presión. Instalación de una tapa superior sobredimensionada para que sirva como tapa de la arqueta de montaje del centro.

Como se puede ver en la figura 3 , el conjunto del transformador queda girado $180^{\circ}$ de manera que las tomas se sitúan en la parte inferior. 


\section{WPSReview International on Sustainable}

\section{Housing and Urban Renewal (RI-SHUR)}
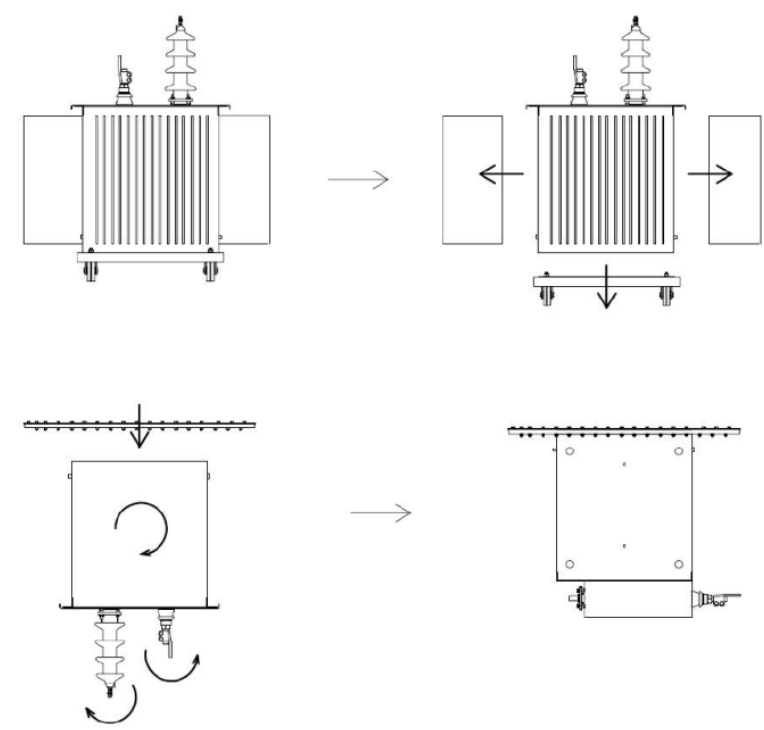

Figura 3: Transferencia desde transformador estándar a transformador propuesto

\subsubsection{Refrigeración del transformador}

Para la correcta evacuación del calor generado en el transformador se dispondrá un intercambiador de calor externo compuesto de tubos horizontales y placas disipadoras de calor. Para el diseño y cálculo de los elementos de este sistema de evacuación de calor se emplean normas europeas (UNE-EN 50216-10, 2010) (UNE-EN 60076-2, 2013) (UNE-EN 60076-7, 2012). El intercambiador se instalará sólidamente anclado en el interior de una carcasa metálica ventilada, sustituyendo parte del bordillo del acerado. De esta forma se consigue que este elemento quede totalmente mimetizado en el entorno y no tenga impacto visual una vez instalado.

La circulación del fluido se realizará mediante bomba circuladora accionada desde la centralita de control (UNE-EN 60296, 2012).

\subsubsection{Sistema de refrigeración alternativa}

Como sistema de refrigeración alternativo al anteriormente propuesto, y para aquellos casos en los que las temperaturas ambientales extremas hagan difícil el correcto intercambio de calor (Gao, Li, Li, 2011), se ha ideado un sistema alternativo a base de máquina frigorífica. En este caso, se dispone intercalado en la parte superior del transformador, una red de tubos que actúa como unidad evaporadora, y una máquina condensadora cuyo serpentín se dispone en el exterior del recinto. El funcionamiento de esta unidad garantiza el perfecto enfriamiento de la máquina e incluso, un subenfriamiento de su temperatura de trabajo que va a propiciar un aumento de la potencia disponible del transformador (Aragüez, 2016b) (Sacchi, Rifaldi, 1989) (UNEIEC/TS 60076-14, 2010). 


\section{WPSReview International on Sustainable \\ Housing and Urban Renewal (RI-SHUR)}

En la figura 4 se comprueba el diseño realizado para el evaporador, de manera que se pueda acoplar fácilmente al transformador propuesto.
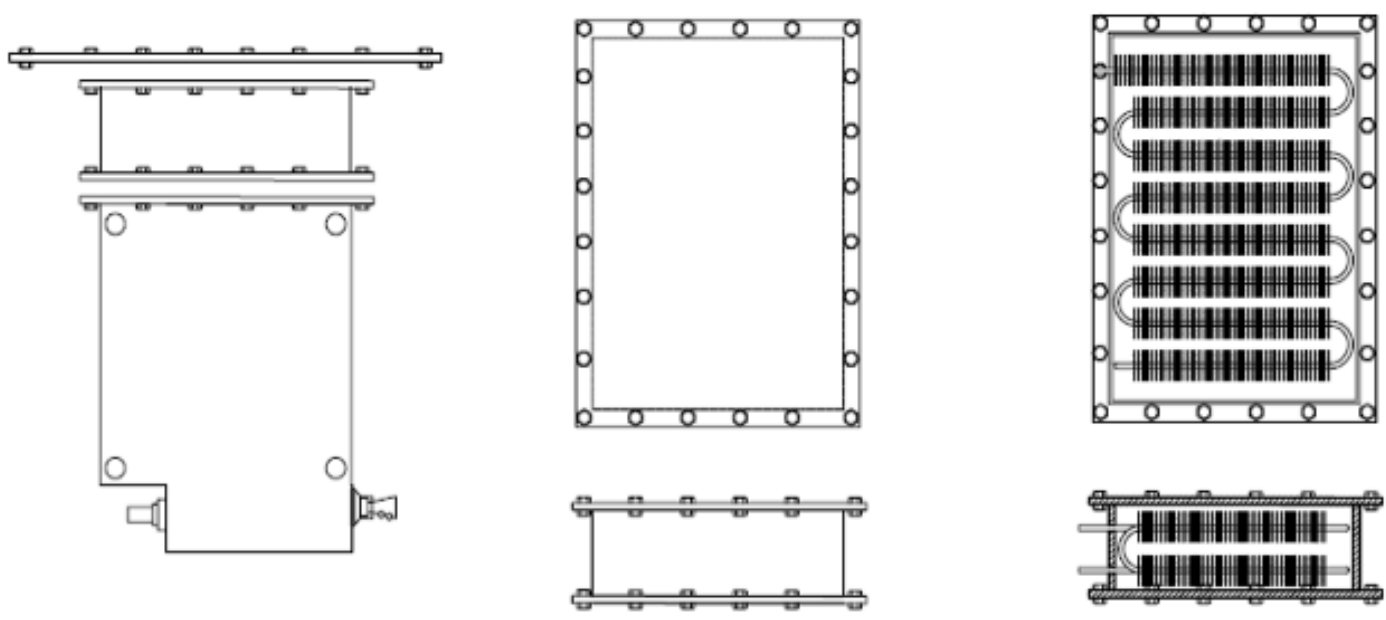

Figura 4: Diseño de nuevo evaporador acoplable al transformador

\subsubsection{Protección contra incendios}

El conjunto se dota de un sistema de protección contra incendios por inundación total de gas inerte. En el interior se disponen las bombonas de gas, las toberas y los detectores de temperatura. En caso de incendios, y de una manera temprana, éste quedaría totalmente sofocado por la acción del gas.

\subsubsection{Centralita de control}

Se prevé un funcionamiento del conjunto totalmente autónomo y una maniobra del aparellaje por accionamiento remoto, es decir, el conexionado o desconexionado de los interruptores de alta y baja tensión se realizará desde la centralita exterior 0 , incluso, desde una central dispuesta en otro lugar diferente, mediante la adopción de la codificación de telegestión de red que cada empresa suministradora de energía eléctrica disponga.

A esta centralita se canalizarán, así mismo, las señales de las sondas de temperatura de la máquina, el estado de disparo del sistema contra incendio, y otras señales que resulten interesantes para la explotación del centro (temperatura ambiente del interior, existencia de vertidos en el fondo de la cuba, etc.). También, esta centralita registrará todas y cada una de las maniobras realizadas, tanto en los disyuntores de alta tensión como en los interruptores automáticos de baja tensión. De esta forma, quedará registrado el elemento accionado, el dato temporal y el nombre o código de la persona 


\section{WPSReview International on Sustainable \\ Housing and Urban Renewal (RI-SHUR)}

o sistema que ha efectuado la orden. Además, se mantendrá un sistema de protocolos de prioridad para cada actuación.

La centralita contará con una pantalla táctil de información y control, además se podrá acceder al sistema desde cualquier dispositivo móvil autorizado con clave de acceso.

Todos estos datos serán gestionados por un sistema big data, con almacenamiento de información en la nube.

En la figura 5 se puede ver, en sección constructiva, el centro de transformación totalmente instalado y la disposición de la centralita de control exterior.

Figura 5: Visita del centro de transformación instalado

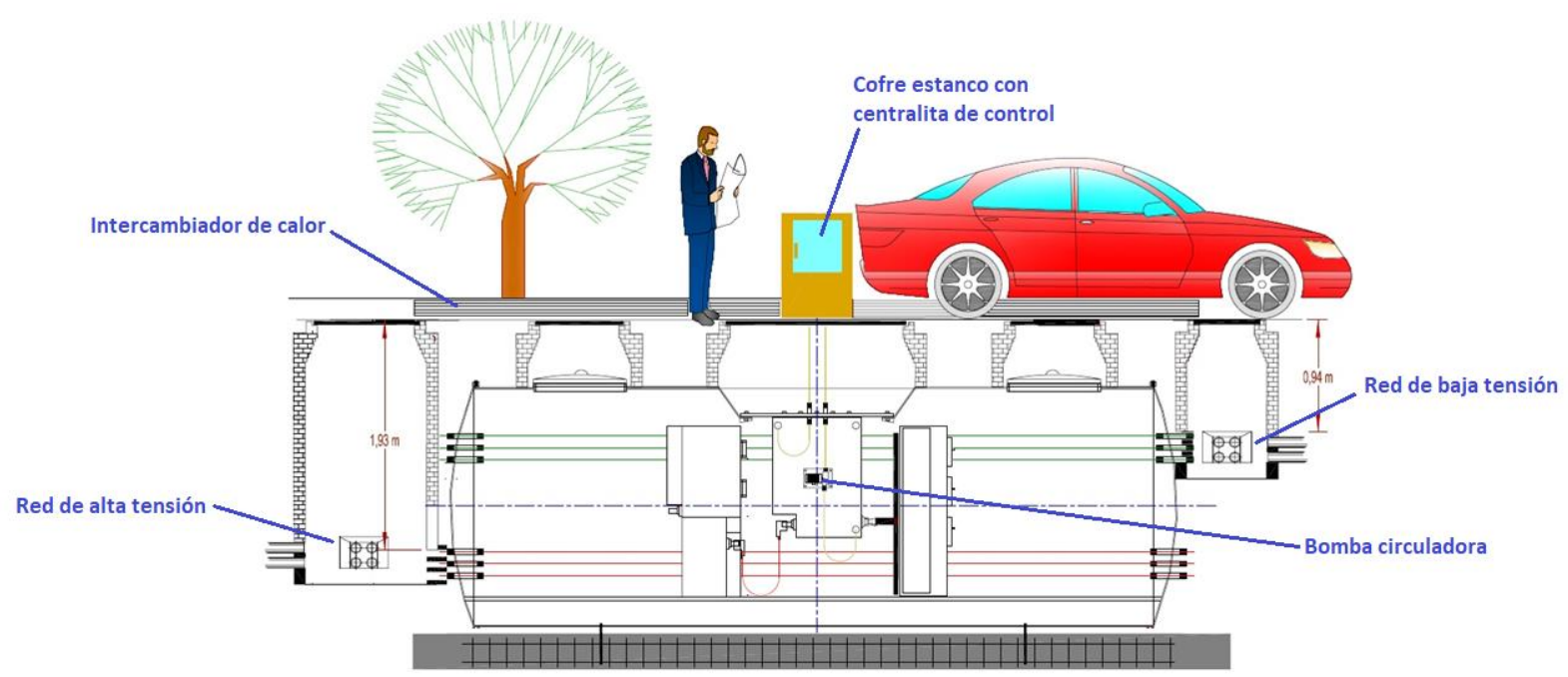

\subsection{Análisis de la propuesta}

El disponer de un diseño totalmente estanco consigue que el centro de transformación pueda seguir operativo incluso en aquellos casos en que, por grandes inundaciones, desbordamientos de ríos, etc. exista un nivel de agua permanente por encima de él. En este caso, el sistema de evacuación de calor sigue funcionando ya que la transferencia de calor en el intercambiador se asegura por el contacto de las placas con el agua (todo el sistema eléctrico queda en el interior del centro que permanece seco y la unidad de control también en su cofre exterior estanco). 


\section{WPSReview International on Sustainable \\ Housing and Urban Renewal (RI-SHUR)}

Esta característica que se ha indicado en el párrafo anterior hace idóneos a estos centros de transformación para funcionar en aquellos países que por su climatología extrema lo hacen muy proclive a soportar grandes inundaciones y desbordamientos de ríos (zonas monzónicas, climas tropicales, ...) y también en aquellos países litorales donde el riesgo de tsunamis es importante. Hay que insistir en que ante estas catástrofes es fundamental mantener el suministro eléctrico para atender a los servicios básicos de seguridad, sanidad, comunicaciones, abastecimiento de agua, etc.

Ante un terremoto, el centro de transformación es estable ya que no tiene estructuras que puedan desmoronarse. Incluso en el caso de desplome de edificios sobre él, el sistema puede seguir operativo ya que la capa de terreno superficial va a amortiguar y minimizar los daños interiores. Además, la estructura cilíndrica de la envolvente hace al sistema especialmente resistente a los esfuerzos que el terreno ejerza sobre él. Esto lo hacen ideal para aquellos países o regiones en las que el índice sísmico es elevado.

El modelo va a tener una afección mínima ante un gran incendio que se produzca en algún inmueble cercano, ya que, por las propiedades aislantes del propio terreno, la temperatura del interior de la instalación no va a sufrir un incremento importante. Por otro lado, ha de tenerse en cuenta que el desarrollo de las llamas es siempre ascendente.

Otra ventaja de este diseño es que la ausencia de rejillas de ventilación impide el acceso de insectos, roedores, polvo, suciedad,... que puede provocar riesgo y accidentes. Además, la estanqueidad del conjunto hace idóneo este sistema para aquellos lugares donde pueda existir una alta concentración de polvo en suspensión (por ejemplo, zonas saharianas, de oriente medio y aquellos lugares donde puedan existir tormentas de arenas que puedan afectar a la red eléctrica).

Como se indica en el apartado 2.5, la paulatina implantación del vehículo eléctrico va a suponer la necesidad de ampliar la potencia disponible en las redes de distribución y por lo tanto, un aumento del número o la potencia de los centros de transformación urbanos. Esto lleva al complejo problema de ubicación de los nuevos centros de transformación. El diseño que se plantea soluciona este inconveniente ya que se ubican las nuevas estaciones bajo los viales u otras zonas públicas. También, como se ha expuesto anteriormente, con este modelo se evita disponer de antiestéticas construcciones en centros urbanos de alto valor turístico u ocupar locales de alto valor económico en zonas comerciales.

Por otro lado, el sistema propuesto permite, por su modularidad que, en caso de demandarse mayores potencias, puedan acoplarse unas unidades a otras para cubrir la demanda necesaria, como se observa en la figura 6. 
WPSReview International on Sustainable

Housing and Urban Renewal (RI-SHUR)
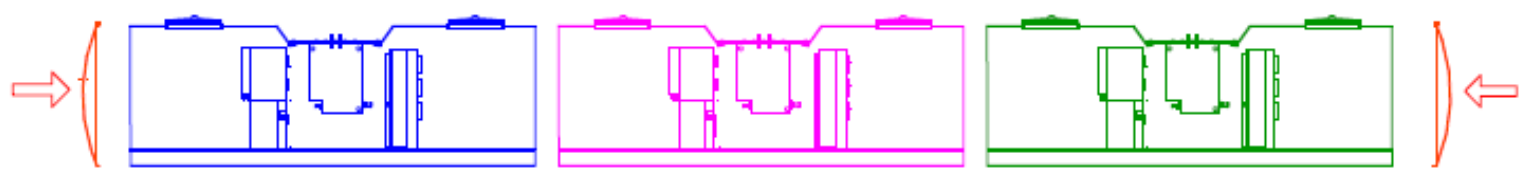

Figura 6: Posibilidad de acoplamiento entre módulos

Como se puede ver en la figura 7, es fácil el montaje del centro de transformación, e incluso la retirada o sustitución de cualquiera de los elementos interiores. Para ello se necesita tan sólo un camión equipado con una pluma. Por otro lado, también es muy fácil sustituir un transformador averiado por otro nuevo o por otro de mayor potencia. En todos estos casos se utiliza la trampilla central de montaje.
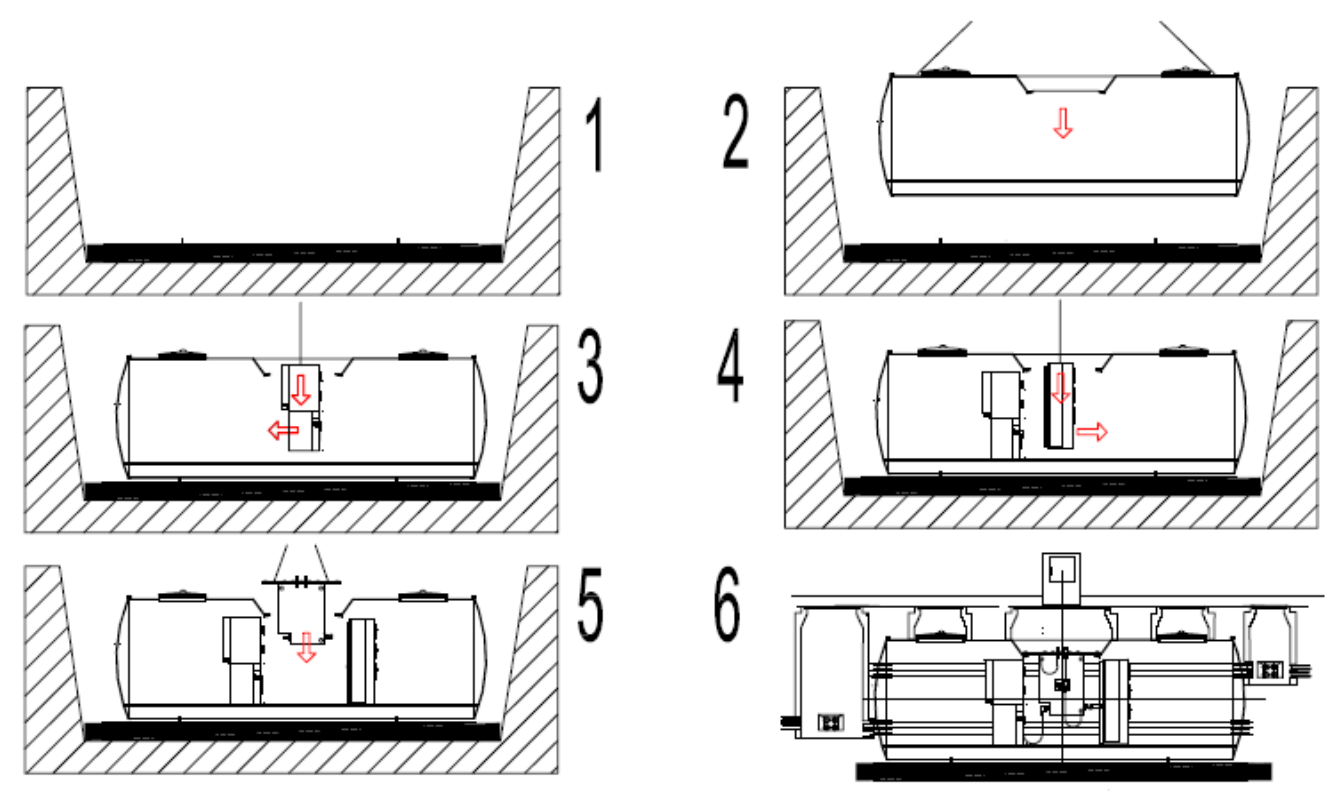

Figura 7: Proceso de puesta en obra del sistema

Por último, la sustitución de los fusibles por los interruptores automáticos presenta una serie de ventajas que se detallan a continuación:

- Aumento de la capacidad de las redes. Posibilidad de ajuste de la intensidad de disparo a la intensidad admisible del cable.

- Repuestos. Con los interruptores automáticos no es necesario mantener un stock de fusibles para reponer los deteriorados.

- Automatización del accionamiento. 


\title{
WPSReview International on Sustainable
}

\author{
Housing and Urban Renewal (RI-SHUR)
}

- Seguridad: El accionamiento desde un lugar remoto hace imposible los accidentes por descargas o quemaduras al accionar interruptores.

- Control: Este tipo de elementos permiten la introducción de las redes de distribución en sistemas de gestión inteligentes (smart grids) desde una central de mando.

- Seguridad contra incendios: El accionamiento desde un lugar externo al centro de transformación permite disponer de un sistema de extinción de incendios por inundación total del recinto.

Como se puede ver, el modelo propuesto presenta una gran cantidad de ventajas frente a los centros existentes, además, la diferencia de precio no es excesiva, del orden de un $20 \%$ más que un centro de transformación subterráneo convencional, sin embargo, las prestaciones son mucho mayores. (Aragüez, 2016b)

\subsection{Conclusiones}

Se puede afirmar que el diseño de los centros de transformación ha variado poco a lo largo de los años, se sigue usando un sistema de ventilación natural mediante rejillas, una máquina transformadora con aletas disipadoras de calor, accionamientos que en la mayoría de los casos son manuales y una envolvente poco resistente ante acciones externas. Si comparamos esta evolución con la existente en cualquier otro sector de bienes de equipo, donde la automatización y la transformación digital ha modificado sustancialmente los productos para hacer que su uso sea más fácil, eficaz e integrable, se puede llegar a la conclusión de que es necesario profundizar en la innovación de los centros de transformación.

Como ya se ha mencionado anteriormente, hay que tener presente lo importante que es mantener el suministro eléctrico, tanto en situaciones de normalidad como ante cualquier catástrofe. Se ha podido constatar, por ejemplo, por las recientes inundaciones en EEUU, que, cuando se produce una catástrofe natural que afecta a las grandes ciudades (inundaciones, terremotos, tsunamis, desbordamientos de ríos, etc.) las infraestructuras y, sobre todo las instalaciones eléctricas de distribución son de las primeras que se ven afectadas. Es importante que el sistema eléctrico quede blindado ante estas situaciones, ya que va a permitir que sigan en uso las infraestructuras críticas para reducir el número de víctimas y conseguir una mayor rapidez en la recuperación de la normalidad.

La innovación que se propone lleva aparejada una mayor seguridad en el uso de las instalaciones, tanto para el personal de mantenimiento como para el resto de ciudadanos, además, la creciente utilización de tecnologías de internet of things en todo lo cotidiano y la incorporación de las smart grids hacen difícil de concebir la existencia de centros de transformación sin que incorporen este tipo de sistemas. 


\section{WPSReview International on Sustainable \\ Housing and Urban Renewal (RI-SHUR)}

Hay que insistir en la necesidad de aumento de potencia eléctrica instalada en un futuro próximo debido a la progresiva implantación de los vehículos eléctricos y que los estándares de seguridad y continuidad de suministro han de ser cada vez más severos.

Se considera que con este innovador modelo de centro de transformación propuesto se contribuye a intentar resolver algunos de los problemas a los que se enfrentan las ciudades del futuro.

\section{4.- Referencias bibliográficas}

ABB (2004). Manual Técnico de Instalaciones Eléctricas. Tomo I: Aparatos de protección y maniobra. Bergamo: ABB SACE.

ABB (2004). Manual Técnico de Instalaciones Eléctricas. Tomo II: La instalación Eléctrica. Bergamo: ABB SACE.

Aragüez del Corral, I. (2016). Centro de transformación modular subterráneo resistente a catástrofes y condiciones adversas. ES 1153658Y.

Aragüez del Corral, I., Aguado Sánchez, J. and Ruiz Aranda, J. (2016). Nuevas tecnologías constructivas en el diseño de centros de transformación de distribución en el medio urbano. Ingeniería Industrial. Universidad de Málaga.

Avelino Pérez, P. (1998). Transformadores de distribución: Teoría, cálculo, construcción y pruebas. México: Reverté.

Capella, R. (2000). Centros de transformación MT/BT. Barcelona: Schneider Electric España S.A.

Gao, M., Li, G. and Li, J. (2011). The temperature dependence of insulation characteristics of transformer oil at low temperatures. Power Engineering and Automation Conference (PEAM), 2011 IEEE.

Real Decreto 223/2008, de 15 de febrero, por el que se aprueban el Reglamento sobre condiciones técnicas y garantías de seguridad en líneas eléctricas de alta tensión y sus instrucciones técnicas complementarias ITC-LAT 01 a 09. I. Disposiciones generales. España.

Real Decreto 337/2014 de 09/05/2014, por el que se aprueban el Reglamento sobre condiciones técnicas y garantías de seguridad en instalaciones eléctricas de alta tensión y sus Instrucciones Técnicas Complementarias ITC-RAT 01 a 23. I. Disposiciones generales. España. 


\section{WPSReview International on Sustainable \\ Housing and Urban Renewal (RI-SHUR)}

Real Decreto 842/2002, de 2 de agosto, por el que se aprueba el Reglamento Electrotécnico de Baja Tensión e Instrucciones Técnicas Complementarias. I. Disposiciones generales. España.

RESCCUE (2018). RESilience to cope with Climate Change in Urban arEas - a multisectorial approach focusing on water. Bristol, Reino Unido: Resccue. Recuperado de http://www.resccue.eu/resccue-project

REE (2018). Simulador de recarga del vehículo eléctrico. Madrid, España: Red Eléctrica de España. Recuperado de http://www.ree.es/sites/all/SimuladorVE/

Sacchi, J. and Rifaldi, A. (1989). Cálculo y diseño de máquinas eléctricas. Buenos Aires: Universidad Nacional de La Plata.

Schneider Electric (2014). El transformador y su entorno, para una protección máxima. (2014). Barcelona: Schneider Electric España S.A.

UNE-EN 50216-10:2010 Accesorios para transformadores de potencia y reactancias. Parte 10: Intercambiadores de calor aceite-aire.

UNE-EN 60296:2012. Fluidos para aplicaciones electrotécnicas. Aceites minerales aislantes nuevos para transformadores y aparamenta de conexión.

UNE-EN 60076-1:2013 Transformadores de potencia. Parte 1: Generalidades.

UNE-EN 60076-2:2013 Transformadores de potencia. Parte 2: Calentamiento de transformadores sumergidos en líquido.

UNE-EN 60076-7:2012 Transformadores de potencia. Parte 7: Guía de carga para transformadores de potencia sumergidos en aceite.

UNE-IEC/TS 60076-14:2010. Transformadores de potencia. Parte 14: Diseño y aplicación de transformadores de potencia sumergidos en líquido aislante utilizando materiales aislantes de alta temperatura 